\title{
Super spreading of oil by condensed water drops
}

\author{
Manoj K. Chaudhury ${ }^{a}$ and Ankur Chaudhury ${ }^{b}$ \\ Received 24th August 2005, Accepted 14th October 2005 \\ First published as an Advance Article on the web 26th October 2005 \\ DOI: 10.1039/b512045b
}

We studied the spreading of a thin film of hexadecane oil on a hydrophobic polystyrene surface, which is partially covered with tiny water droplets. In spite of the fact that the spreading coefficient of hexadecane is slightly negative on water, the oil actually spreads faster on the droplet covered polystyrene than on the pure substrate. The observed effect is partly due to the geometric factors that lead to greater reduction of oil-substrate interfacial energy in comparison to the increase of oil-vapor surface energy. However, it is more important to consider the reduction of the resistance to fluid motion in the presence of condensed droplets. We provide an explanation of the phenomenon by drawing an analogy to the spreading on a rough surface, in which each water drop nucleates and reinitiates the spreading process. The phenomenon is therefore an approximate 2D version of liquid invasion through porous media.

We observed that hexadecane oil spreads faster on a hydrophobic polystyrene surface, if the latter is partially covered with condensed water droplets. Based on their relative surface free energies, the spreading coefficient $(S)$ of hexadecane (surface tension $27.7 \mathrm{mN} \mathrm{m}^{-1}$ ) should be positive on polystyrene (surface free energy $\sim 36 \mathrm{~mJ} \mathrm{~m}^{-2}$ ) and negative on water ${ }^{1}$ (dispersion component of surface tension $\sim 22 \mathrm{mN} \mathrm{m}^{-1}$ ). Thus from the surface energy point of view there is intrinsically no greater driving force for hexadecane to spread on a droplet-covered polystyrene than on the pure polymer. The phenomenon may be partially understood by considering the fact that the increase of the free energy of adhesion between the oil and the droplet-covered substrate is greater than the decrease of the cohesive energy of oil during the creation of new oil-vapor surface area. More importantly, the condensed water droplets enhance the spreading rate by nucleating and re-initiating the spreading oil like the asperities of a rough surface. ${ }^{2-5}$

We performed the following experiments. Water vapor emanating from a warm $\left(48{ }^{\circ} \mathrm{C}\right)$ water source was allowed to condense on a polystyrene Petri dish (Fisher Brand, $100 \mathrm{~mm} \times 15 \mathrm{~mm}$ ) at room temperature for $10 \mathrm{~s}$. This resulted in $\sim 44 \%$ coverage of the Petri dish by water droplets of sizes in the range of 5-10 $\mu \mathrm{m}$ (Fig. 1). A similar Petri dish devoid of such condensed drops was used as a control. The liquid drops, as illuminated with diffused white light, were observed with a standard video camera and recorded with a VCR for analysis at leisure. After placing a small drop of hexadecane on a polystyrene substrate, it was quickly covered with another polystyrene cover to minimize evaporation

${ }^{a}$ Department of Chemical Engineering, Lehigh University, Bethlehem,

PA 18015, USA.E-mail: mkc4@lehigh.edu

${ }^{b}$ Cornell University, Ithaca, NY 14853, USA of water. ${ }^{6}$ Fig. 2 shows that hexadecane spreads faster on the surface covered with condensed water drops than on the bare surface. In order to ascertain the power law of spreading in both cases, the spreading radii were normalized as $r / V^{1 / 3}(V=$ volume) and plotted against time on a logarithmic plot (Fig. 3). The spreading exponent for hexadecane on polystyrene is thus found to be about 0.14 during its active stage of spreading, which is similar to the values $(0.1-0.14)$ reported in literature ${ }^{2,3,5,7-11}$ for liquids spreading on smooth surfaces. However, after 2 min, the spreading slowed down dramatically. The spreading kinetics of hexadecane on the condensed water drops, on the other hand, exhibited a

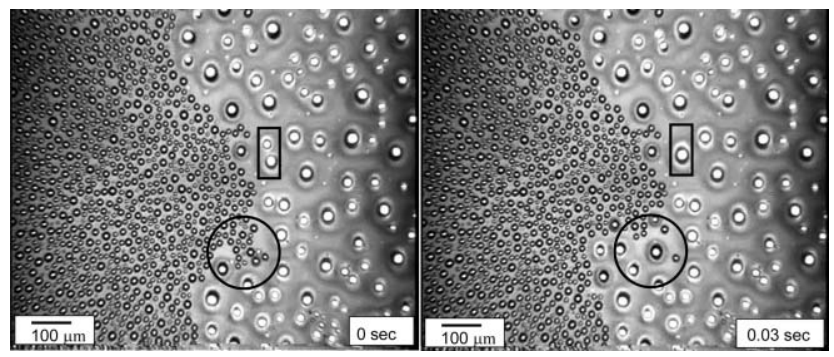

Fig. 1 Video snapshots of the spreading of hexadecane on a polystyrene surface partially covered with condensed water droplets. Spreading occurs from right to left of the figure. Note that the water drops behind the spreading front are larger than those lying ahead. This results from the migration of water drops towards each other and their subsequent coalescence. These coalescence events can be seen clearly within the regions marked as rectangles and circles. In the first case, two drops merge to become one. In the second case several drops coalesce.

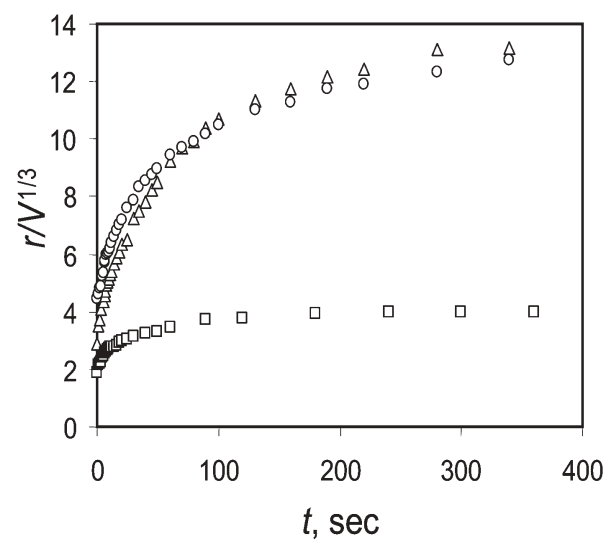

Fig. 2 Spreading kinetics of a $3 \mu \mathrm{l}$ drop of hexadecane on a polystyrene Petri dish. Squares indicate the control experiment, the circles indicate a polystyrene surface covered with condensed water drops and the triangle indicates a polystyrene surface covered with $\sim 1 \mu \mathrm{m}$ size glass beads (see below). 


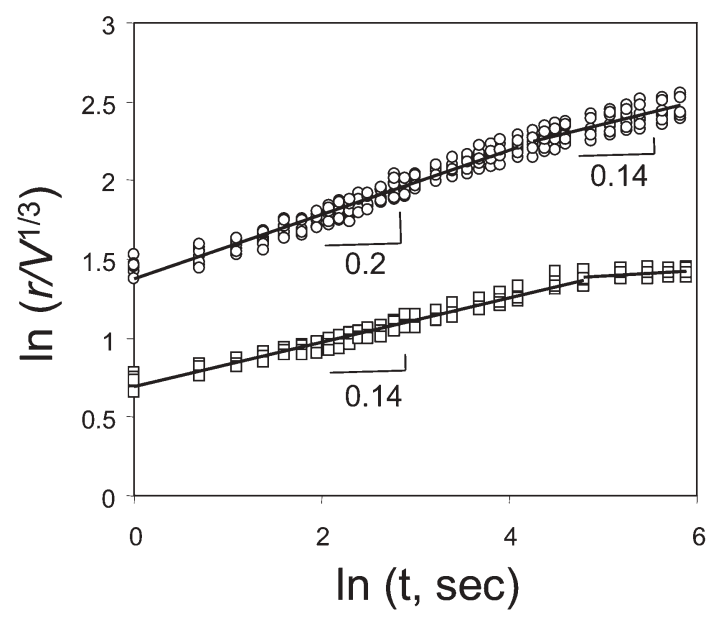

Fig. 3 Logarithmic plots of the spreading kinetic data of hexadecane on polystyrene Petri dishes. Squares indicate the control experiment and the circles indicate the experiments on condensed water droplets. For each surface, hexadecane drops of sizes $2 \mu \mathrm{l}, 3 \mu \mathrm{l}$ and $4 \mu \mathrm{l}$ and their duplicates were used.

power law of 0.2 for first $2 \mathrm{~min}$ followed by the decrease of the exponent to 0.14 . The observed exponent during the active stage of spreading is within the range of the reported values ${ }^{2,3}$ of the spreading exponents $(0.2-0.5)$ of liquids on rough surfaces. It was interesting to observe that the water drops, which are already wetted by the hexadecane film, are quickly dislodged from the polystyrene surface. The dislodged drops move towards each other in a somewhat random fashion and coalesce to form larger drops of approximately $20-30 \mu \mathrm{m}$ (Fig. 1). This observation suggests that negative pressure regions exist on the wetting film between the drops due to capillary bridging of water drops by hexadecane film, which induce droplet migration. The pressure field is inhomogeneous because of the randomness of the droplet sizes and their spacings. Existence of this negative pressure region near the wetting edge seems to be what drives the spreading process as well.

Next we attempt to derive an approximate expression for the spreading kinetics by adopting an approach similar to that developed by Marmur ${ }^{12}$ to account for the capillary invasion of liquid in a porous medium. The method is also related to that of Wenzel $^{13}$ in accounting for the spreading of liquids on rough surfaces. Fig. 4 shows a layer of liquid of average height $h$ and radius $r$ spreading on a polystyrene surface covered with condensed water drops. We start with the change in the total interfacial energy due to spreading as follows:

$$
\mathrm{d} U=\gamma_{\mathrm{sl}} \mathrm{d} A_{\mathrm{sl}}+\gamma_{\mathrm{sv}} \mathrm{d} A_{\mathrm{sv}}+\gamma_{\mathrm{lv}} \mathrm{d} A_{\mathrm{lv}}
$$

where, $\gamma_{\mathrm{sl}}, \gamma_{\mathrm{sv}}$ and $\gamma_{\mathrm{lv}}$ indicate the surface tension of the solidliquid, solid-vapor and liquid-vapor interfaces; $A_{\mathrm{sl}}, A_{\mathrm{sv}}$ and $A_{\mathrm{lv}}$ are the corresponding surface areas. In our case, when the hexadecane

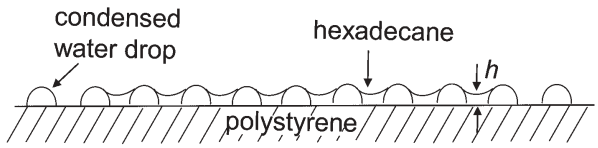

Fig. 4 A schematic showing the spreading of hexadecane oil on a polystyrene surface covered with water drops. oil (o) film spreads over water (w) droplet covered polystyrene (p) surface, there are two possibilities to consider. If the oil-vapor interface of the spreading film does not roughen significantly, $\mathrm{d} U$ assumes the following form:

$$
\begin{gathered}
\mathrm{d} U=\left(\gamma_{\mathrm{ow}}-\gamma_{\mathrm{wv}}\right) \phi \beta(2 \pi r \mathrm{~d} r)+\left(\gamma_{\mathrm{op}}-\gamma_{\mathrm{pv}}\right)(1-\phi)(2 \pi r \mathrm{~d} r)+ \\
\gamma_{\mathrm{ov}}(2 \pi r \mathrm{~d} r)
\end{gathered}
$$

where, $\phi$ is the fractional area of the surface covered with the water drops, $\beta$ is the ratio of the surface area of a drop to its projection on the surface, which is close to 2 since the contact angle of water on polystyrene is $\sim 90^{\circ}$. In deriving eqn. (2), we assumed that most part of a water droplet is wetted by hexadecane. We feel that this assumption is justified because the thickness of the oil film is comparable to the size of the drops and the contact angle of hexadecane on water is reasonably low $\left(\sim 30^{\circ}\right.$, using Neuman triangle). The driving force for spreading can be obtained as $F=-\mathrm{d} U / \mathrm{d} r$, i.e.

$$
F=-\frac{\mathrm{d} U}{\mathrm{~d} r}=2 \pi r\left[S_{\mathrm{ow}} \phi \beta+S_{\mathrm{op}}(1-\phi)+\gamma_{\mathrm{ov}}(\beta-1) \phi\right]
$$

Where, $S_{\mathrm{ij}}$ is the spreading coefficient, which is related to the interfacial tensions as,

$$
S_{\mathrm{ij}}=-\gamma_{\mathrm{ij}}-\gamma_{\mathrm{iv}}+\gamma_{\mathrm{jv}}
$$

However, as the hexadecane continues to spread on the rough surface, it thins and consequently the oil-vapor interface roughens considerably. With an appreciably roughened oil-vapor interface, the driving force for spreading reduces to that shown in eqn. (4).

$$
F=2 \pi r\left[S_{\text {ow }} \phi \beta+S_{\text {op }}(1-\phi)+\gamma_{\text {ov }}\left(\beta-\beta^{*}\right) \phi\right]
$$

Where, $\beta^{*}$ is a parameter representing the roughness of the oilvapor interface. Its value in the beginning of the spreading should be closer (but not equal) to unity. However, at the late stage of spreading, its value could approach $\beta$. There are several spreading situations to consider.

1. $S_{\text {ow }}$ and $S_{\text {op }}$ are both positive. In this case, the oil film spreads on the substrate unconditionally. Nevertheless, the spreading should slow down as the film becomes thin enough so that the oilvapor interface roughens considerably $\left(\beta^{*} \rightarrow \beta\right)$. As the oil film thins faster on a surface covered with larger drops, the transition from fast to slow spreading is expected to occur earlier than that with the finer drops.

2. Limited spreading occurs if $S_{\mathrm{ow}} \phi \beta+S_{\mathrm{op}}(1-\phi)$ is strongly negative and its absolute magnitude is larger than $\gamma_{\text {ov }}\left(\beta-\beta^{*}\right)$ for all values $\beta^{*}$.

3. $S_{\text {ow }}$ and $S_{\text {op }}$ are both negative, or of opposite signs, so that $S_{\mathrm{ow}} \phi \beta+S_{\mathrm{op}}(1-\phi)$ is slightly negative. If the absolute magnitude of $S_{\mathrm{ow}} \phi \beta+S_{\mathrm{op}}(1-\phi)$ is less than $\gamma_{\mathrm{ov}}(\beta-1)$ spreading should occur. However, as the oil-vapor interface continues to roughen $\beta^{*}$ approaches $\beta$, when spreading slows down and ultimately stops. This is indeed consistent with the prediction of the Wenzel equation $^{13,14}$ that a liquid, the intrinsic contact angle of which is less than $90^{\circ}$ on a surface, would spread even more on the latter if it is roughened. ${ }^{15}$

In our case, about $44 \%(\phi=0.44)$ of the polystyrene surface is covered with water drops. Using the known values of the surface 
tensions of water, hexadecane and polystyrene, we estimate $S_{\text {ow }} \sim-6 \mathrm{mN} \mathrm{m}^{-1}$ and $S_{\mathrm{op}} \sim 8 \mathrm{mN} \mathrm{m}^{-1} . S_{\mathrm{ow}} \phi \beta+S_{\mathrm{op}}(1-\phi)$ is thus estimated to be about $-0.8 \mathrm{mN} \mathrm{m}^{-1}$. In the beginning of spreading, the roughening of the oil-vapor interface is negligible (i.e., $\beta^{*}$ is slightly larger than unity), we have the value of the effective spreading coefficient $S_{\text {ow }} \phi \beta+S_{\text {op }}(1-\phi)+\gamma_{\text {ov }}(\beta-1) \phi$ close to $11 \mathrm{mN} \mathrm{m}^{-1}$. Slight roughening of the oil-vapor interface (due to capillary bridging effect) is necessary in order to create a pressure gradient in the hexadecane film for it to spread outward. This is consistent with the lateral migration and coalescence of the water drops observed experimentally near the contact line region (Fig. 1). This situation is described by eqn. (4) with $1<\beta^{*}<\beta$, which would lead to an overall spreading coefficient $S_{\text {ow }} \phi \beta+$ $S_{\text {op }}(1-\phi)+\gamma_{\text {ov }}\left(\beta-\beta^{*}\right) \phi$ less than $11 \mathrm{mN} \mathrm{m}^{-1}$, but its value should still be positive and may not be appreciably different from $S_{\text {op }}$. In summary, the presence of the water drops on the polystyrene surface does not decrease the spreading co-efficient in any remarkable manner, and that it remains positive. We thus need to look into the possible reduction of the resistance of fluid motion by the water drops as the possible mechanism for fast spreading.

The reduction of the resistance to the fluid motion may be understood as follows. When a small liquid drop of radius $r$ and viscosity $\mu$ spreads on a flat surface, the viscous dissipation ${ }^{10}$ is concentrated at the wedge that gives rise to a drag force $\left(F_{1}\right) \sim$ $\mu r \bar{v} \alpha / \theta$; here, $\theta$ is the dynamic contact angle, $\bar{v}$ is the average velocity of the drop, and $\alpha$ is a logarithmic prefactor $(\sim 10)$ needed to prevent the divergence of shear stress at the contact line.

The magnitude of $F_{1}$, however, decreases as $\theta$ increases, which results from the accumulation of the spreading liquid under asperities or condensed drops. The liquid with a temporary high dynamic contact angle would, nevertheless, tend to relax to a lower value. However, if the contact line reaches from one droplet to another, before $\theta$ relaxes to a lower value, the resistance to the fluid motion could be reduced. This effect can be demonstrated in a very simple experiment, in which several small $(\sim 1 \mathrm{~mm})$ water drops are placed at regular intervals along a straight line on a polystyrene surface. When the drops are reasonably close to each other $(\sim 1 \mathrm{~mm})$ and a small hexadecane drop is placed near one end of the array, it spreads spontaneously from one end to the other with considerable speed. Video-microscopic observation shows the process of accumulation of oil under each drop, respreading with finite dynamic contact angle, re-accumulation of oil in the second drop and so on. The speed of spreading of hexadecane oil through this array of drops can be controlled by their distance of separation. When the separation distance is larger than $\sim 2 \mathrm{~mm}$, spreading slows down and eventually stops. We found that this effect can not only be created with water drops, with which hexadecane exhibits a finite contact angle, but also with glycerin drops where spreading coefficient is positive.

As the wedge dissipation begins to play a lesser role, the hydrodynamic force $\left(F_{2}\right)$ resisting the drop motion is due to the integrated shear stress over the entire film, which is $\mu \bar{v} r^{2} / h$, where $h$ is the thickness of the film (or drop). The relative magnitudes of the two forces can be judged by looking at the ratio:

$$
\frac{F_{1}}{F_{2}} \approx \frac{\alpha h}{r \theta} \text {. }
$$

As the drop continues to thin, the ratio $h / r$ decreases, which, in our case, easily attains a value of $\sim 10^{-3}$ within $10-15 \mathrm{~s}$ of spreading. Such a low aspect ratio of the drop coupled with the fact that $\theta$ is substantially greater than zero make the contribution of $F_{1}$ significantly less than $F_{2}$. We thus take the total energy dissipation to be distributed over the entire film as follows:

$$
F \bar{v}=\mu \int\left(\frac{\partial v}{\partial z}\right)^{2} \mathrm{~d} V \sim \mu\left(\frac{\bar{v}}{h}\right)^{2}\left(\pi r^{2} h\right) \phi_{\mathrm{V}}
$$

where $\partial v / \partial z$ is the velocity gradient in the liquid, which is on the order of $\bar{v} / h . \phi_{\mathrm{V}}$ is the volume fraction of the spread film that is pure oil, which is approximately equal to $1-\phi$. The above simplification ignores the events associated with the dislodging and migration of water drops, which are energy dissipative processes as well. Eqns. (4) and (5) can be combined to yield eqn. (6).

$$
\bar{v}=\frac{\mathrm{d} r}{\mathrm{~d} t} \approx \frac{2\left(S_{\mathrm{op}}(1-\phi)+S_{\mathrm{ow}} \phi \beta+\gamma_{\mathrm{ov}}\left(\beta-\beta^{*}\right) \phi\right) h}{\mu \phi_{\mathrm{v}} r}
$$

There are two situations to consider. In the first place, the average thickness of the film does not vary significantly. In that case, eqn. (6) can be integrated to yield the radius of the drop increasing as $t^{1 / 2}$, i.e.,

$$
r \approx\left[\frac{\left(S_{\mathrm{op}}(1-\phi)+S_{\mathrm{ow}} \phi \beta+\gamma_{\mathrm{ov}}\left(\beta-\beta^{*}\right) \phi\right) h t}{\mu(1-\phi)^{2}}\right]^{\frac{1}{2}}
$$

For the oil film to spread with constant $h$, however, an unlimited source of liquid is required. In our case, the source of liquid is finite. Close observation of the spreading process shows that a liquid film of diameter several times larger than that of the deposited liquid spreads out of the main liquid drop within seconds after its deposition, much like that of a precursor film. The small amount of liquid initially remaining at the center of the film then gets sucked into the fast spreading film and the spread film takes the shape of a pancake. Thus considering the film to spread with constant volume, we use the constraint $\pi r^{2} h \phi_{\mathrm{V}}=V$. Eqn. (6) can thus be integrated to obtain the following result:

$$
r \approx\left[\frac{\left(S_{\mathrm{op}}(1-\phi)+S_{\mathrm{ow}} \phi \beta+\gamma_{\mathrm{ov}}\left(\beta-\beta^{*}\right) \phi\right) V t}{\mu(1-\phi)^{2}}\right]^{\frac{1}{4}}
$$

Note that the theoretically obtained spreading exponent $(0.25)$ is slightly higher than that (0.2) obtained experimentally. A different scaling law is obtained by considering the flow to be occurring through equivalent porous channels. In this case the thin film equation is:

$$
\bar{v}=\frac{\mathrm{d} r}{\mathrm{~d} t} \approx \frac{h^{2} \Delta P}{\mu r}
$$

where the pressure drop $\Delta P$ is controlled by the spacing $(R)$ between the condensed drops and their sizes. For a drop spreading with constant volume, eqn. (9) can be integrated to obtain the following result:

$$
r \approx\left(\frac{\gamma V^{2} t}{\mu R}\right)^{1 / 6}
$$

Eqn. (10) predicts a spreading exponent of $\sim 0.17$ which is slightly lower than the experimental observation. However, the assumption that $\Delta P$ is independent of the film thickness $h$ is 
flawed. If $\Delta P$ varies inversely with $h$, we would recover the same scaling as that shown in eqn. (8).

As mentioned above, we have ignored all the details involving the coalescence of the drops and the corresponding surface energetics. Coalescence of the water drops in the thin oil film involves the reductions of the oil-water and the water-polymer interfacial free energies and the corresponding increase of the polymer-oil interfacial free energy. By carrying out the contributions of these processes to the total energy ( $U_{1}$, eqn. (11)), the resultant additional force $\left(F_{1}\right)$ is given by eqn. (12):

$$
\begin{gathered}
\mathrm{d} U_{1}=\phi\left(1-\frac{R_{1}}{R_{2}}\right)\left(\gamma_{\mathrm{po}}-\gamma_{\mathrm{wp}}-\beta \gamma_{\mathrm{ow}}\right)(2 \pi r \mathrm{~d} r) \\
F_{1}=2 \pi \phi\left(1-\frac{R_{1}}{R_{2}}\right)\left(\beta \gamma_{\mathrm{ow}}+\gamma_{\mathrm{wp}}-\gamma_{\mathrm{po}}\right) r
\end{gathered}
$$

Where, $R_{1}$ and $R_{2}$ are the base radii of the water droplets before and after coalescence. Since, $R_{1}<R_{2}$ and $\beta \gamma_{\mathrm{ow}}+\gamma_{\mathrm{wp}}$ is expected to be larger than $\gamma_{\text {po }}$; thus, we anticipate that the coalescence would enhance the driving force. Eqn. (12), in conjunction with eqns. (3) or (4), poses an interesting scenario when $F=0$, but $F_{1}>0$. The film could still keep on spreading on the water drop-covered polymer surface fuelled by the energy of coalescence. Nevertheless, the coalescence needs to be triggered by some other external force and a large fraction of this energy will be lost in the coalescence process itself.

The energy dissipation in and around the water drops has two components: (1) a steady dissipation of energy in and around the drops due to the transfer of shear stress from the oil film that lead to a Hadamard flow ${ }^{16}$ and (2) a dissipation due to the coalescence of the drops. We expect the dissipation related to the Hadamard flow to scale as

$$
\sim \Sigma \frac{1+1.5 \chi}{1+\chi} \mu a \bar{v}^{2} r^{2}
$$

, where $\chi$ is the ratio of the viscosities of water and hexadecane and $\Sigma\left(\sim \phi / a^{2}\right)$ denotes the numbers of drops per unit area. The ratio of this dissipation to that in the hexadecane film (eqn. (5)) is $\sim h / a$. Although the thickness of hexadecane initially is similar to the size of the condensed water droplets (i.e., $h \approx a$ ), the ratio $h / a$ becomes smaller than unity after the droplets coalesce and grow. Thus, we feel that although the dissipation in and around the droplets cannot be neglected, it may not constitute a large fraction of the overall dissipation. The estimation of the dissipation due to coalescence is more complicated as the process is, at first, dominated by inertia and then by viscous forces. A rough estimate of this dissipation in the viscosity dominated regime ${ }^{17}$ is due to $\sim \Sigma \mu_{\mathrm{w}} a V_{\mathrm{ca}}^{2} r^{2}$, where $\mu_{\mathrm{w}}$ is the viscosity of water and $V_{\mathrm{ca}}$ is the capillary velocity. Although this dissipation is significantly large, its source is contained in the interfacial energy terms shown in eqn. (11). Hence, the formulations leading up to eqn. (8) should remain nearly unchanged.

To re-iterate, we found that the fast spreading of hexadecane on polystyrene covered with tiny water droplets is very much similar to spreading induced by surface roughness. Indeed, the spreading kinetics of hexadecane on these water drop covered polystyrene surfaces is similar to that seen with a Petri-dish covered partially with $\sim 1 \mu \mathrm{m}$ size glass beads (Fig. 2). In the latter case, the spreading exponent $(0.27)$ is somewhat higher than that $(0.2)$

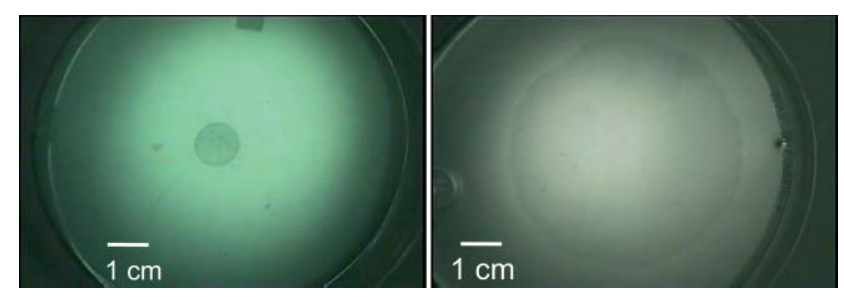

Fig. 5 Preparation of thin polymer film using condensation assisted wetting. A $30 \mu \mathrm{l}$ drop of octane containing $10 \%$ of cross-linkable PDMS was deposited on pure polystyrene (left) and that (right) covered with condensed water drops. The oil drop spreads to a much larger diameter in the presence of condensed droplets. After the octane evaporates, the polymer cross-links at room temperature overnight. During this time the water droplets evaporate as well. This procedure produced a cross-linked PDMS film of $5.4 \mathrm{~cm}$ diameter and $\sim 1.5 \mu \mathrm{m}$ thick (right). Films of variable thickness and diameter can be produced by controlling the concentration of PDMS as well as choosing the career liquid of appropriate viscosity, surface tension and vapor pressure.

observed with condensed water drops but closer to the theoretical estimate (0.25). A complete analysis of these kinds of studies involving particles of various sizes will be published separately.

Droplet-induced enhancement of spreading rate of liquids could have practical applications in some situations where surfactants cannot be used. In due course of time, these droplets do disappear in the thin liquid film by dissolution and evaporation, even in and through such non-polar liquids as hexadecane or silicone oil. This phenomenon may therefore be utilized to produce thin films of controllable thickness and diameter (Fig. 5).

The results reported in this paper may also be relevant to the interpretations of the wetting kinetics of liquids on surfaces that are conducive to the nucleation of water droplets. One such example $^{18}$ is the spreading of aqueous solutions of silicone surfactants (super-wetters) on a hydrophobic surface, which is enhanced by high humidity. Lin et al. ${ }^{19}$ found evidence of the presence of small condensed water drops on hydrophobic surfaces when such super-spreading occurs. Although the situation with the surfactant drop is strongly influenced by the surface tension gradient, ${ }^{20}$ the free energy reduction due to the coalescence of the condensed water drops with the primary wetting film as well as maintenance of the dynamic contact angle to a reasonably high value may play important roles in this class of spreading as well.

\section{Note added in proof}

Derivation of eqn. (3) (and (4)) is based on the assumption that the water droplets do not change their shapes even when they are wetted by hexadecane. This assumption is justified if the oil drops are virtually pinned to the substrate due to contact angle hysteresis. Assuming full relaxation of the contact line, we get the following effective spreading coefficient:

$$
\begin{gathered}
S=\left(\phi_{1}-\phi_{2}\right) \gamma_{\mathrm{pw}}+\left(\phi_{1} \beta_{1} \gamma_{\mathrm{wv}}-\phi_{2} \beta_{21} \gamma_{\mathrm{ow}}\right)+\left(1-\phi_{1}\right)- \\
\left(1-\phi_{2}\right) \gamma_{\mathrm{op}}-\beta^{*} \gamma_{\mathrm{ov}}
\end{gathered}
$$

where the $\phi_{1}$ and $\phi_{2}$ are the fractions of the surface occupied by water drops before and after being wet by oil. $\beta_{1}$ and $\beta_{2}$ are the corresponding roughness parameters of the water drops. All of the above parameters can be calculated from the contact angles of water on polystyrene in air and oil. Numerical estimates show that 
$S$ can reach about $18 \mathrm{mN} \mathrm{m}^{-1}$ if $\beta^{*} \rightarrow 1$, which is slightly higher than that $\left(11 \mathrm{mN} \mathrm{m}^{-1}\right)$ obtained from eqn. (3). In reality, as the contact lines would be partially relaxed due to wetting hysteresis, a value of $S$ is expected to be between $11 \mathrm{mN} \mathrm{m}^{-1}$ and $18 \mathrm{mN} \mathrm{m}^{-1}$, which would be further reduced due to some roughening of the oil-vapor interface.

One of the reviewers of this paper pointed out two important references, ${ }^{21,22}$ which are relevant to the present report. Ref. 21 reports fast spreading of low surface tension liquids on microtextured surfaces. In this case, as the liquid source is virtually unlimited, the spreading exponent is about 0.5 , which is consistent with eqn. (7) of this paper. Ref. 22 reports various scenarios of the improved wetting of liquids by particles. The same authors also report ${ }^{23}$ that particles can enhance wetting by effectively reducing the surface energy of liquid-vapor and liquid-liquid interfaces.

\section{References}

1 C. J. van Oss, L. K. Ju, M. K. Chaudhury and R. J. Good, J. Colloid Interface Sci., 1989, 128, 313.

2 W. D. Bascom, R. L. Cottington and C. R. Singleterry, Contact Angle, Wettability and Adhesion, Adv. Chem. Ser. 43, American Chemical Socierty, Washington, DC, 1964, p. 355.

3 A. M. Cazabat and M. A. Cohen Stuart, J. Phys. Chem., 1986, 90, 22, 5845.

4 J. A. Mann, L. Romero, R. R. Rye and F. G. Yost, Phys. Rev. E: Stat. Phys., Plasmas, Fluids, Relat. Interdiscip. Top., 1995, 52, 4, 3967.

5 C. M. Lin, R. M. Ybarra and P. Neogi, Adv. Colloid Interface Sci., 1996, 67, 185.

6 The spreading studies with hexadecane on polystyrene were carried out under ambient condition at $\sim 50 \%$ RH by enclosing the polystyrene dish with another polystyrene cover, which reduced the evaporation of droplets significantly. The spreading studies on the condensed water drops were carried out by placing the drop on the surface and inverting this substrate against another dish filled with water at room temperature. This ensured negligible evaporation of water during the duration of the experiments. Hexadecane was purified by passing it through neutral grade alumina.

7 L. H. Tanner, J. Phys. D: Appl. Phys., 1979, 12, 1473.

8 A. Marmur, Colloids Surf., A, 2004, 250, 409.

9 A. Marmur, Adv. Colloid Interface Sci., 1983, 19, 75.

10 P. G. de Gennes, Rev. Mod. Phys., 1985, 57, 3, 82.

11 J. Lopez, C. A. Miller and E. Ruckenstein, J. Colloid Interface Sci, 1976, 56, 460.

12 A. Marmur, Langmuir, 2003, 19, 5956.

13 R. N. Wenzel, Ind. Eng. Chem., 1936, 28, 988.

14 R. J. Good, M. K. Chaudhury and C. Yeung, in First International Congress on Adhesion Science and Technology: Mittal Festschrift, ed. W. J. Van Ooij and H. R. Anderson, VSP, Netherlands, 1998, pp. 181-197.

15 We have directly verified this prediction with a polydimethyl siloxane elastomer crosslinked in contact with an aluminum surface that was roughened by a grit blasting procedure. A smooth PDMS elastomer is not wettable by isopropyl alcohol (IPA) or hexadecane. However, when roughened using the above procedure, these liquids spread on it spontaneously. An earlier observation of a similar effect was reported in ref. 14.

16 J. S. Hadamard, C. R. Acad. Sci. Paris, 1911, 152, 1735.

17 J. Frenkel, J. Phys. (Moscow), 1945, 9, 385

18 R. M. Hill, Curr. Opin. Colloid Interface Sci., 1998, 3, 247.

19 Z. Lin, R. M. Hill, H. T. Davis and M. D. Ward, Langmuir, 1994, 10, 4060.

20 A. D. Nikolov, D. T. Wasan, A. Chengara, K. Koczo, G. A. Policello and I. Kolossvary, Adv. Colloid Interface Sci., 2002, 96, 1-3, 325.

21 J. Bico, C. Tordeux and D. Quere, Europhys. Lett., 2001, 55, 214.

22 W. A. Goedel, Europhys. Lett., 2003, 62, 607.

23 H. Xu and W. A. Goedel, Langmuir, 2003, 19, 4950 